\title{
Limits of the trivial bundle on a curve
}

\author{
Arnaud Beauville
}

\begin{abstract}
We attempt to describe the vector bundles on a curve $C$ which are specializations of $\mathcal{O}_{C}^{2}$. We get a complete classification when $C$ is Brill-Noether-Petri general, or when it is hyperelliptic; in both cases all limit vector bundles are decomposable. We give examples of indecomposable limit bundles for some special curves.
\end{abstract}

Keywords. Vector bundles; limits; Brill-Noether theory; hyperelliptic curves

2010 Mathematics Subject Classification. 14H60

\section{[Français]}

\section{Titre. Limites du fibré trivial sur une courbe}

Résumé. Nous essayons de décrire les fibrés vectoriels qui sont des spécialisations de $\mathcal{O}_{C}^{2}$. Nous obtenons une classification complète lorsque $C$ est générale au sens de Brill-Noether-Petri, ou lorsque $C$ est hyperelliptique; les fibrés limites sont décomposables dans chacune des deux situations. Nous donnons également des exemples de fibrés limites indécomposables sur certaines courbe spéciales.

Received by the Editors on March 8, 2018, and in final form on August 30, 2018. Accepted on October 4, 2018.

Arnaud Beauville Université Côte d'Azur, CNRS - Laboratoire J.-A. Dieudonné, Parc Valrose, F-06108 Nice cedex 2, France e-mail: arnaud.beauville@unice.fr 


\section{Contents}

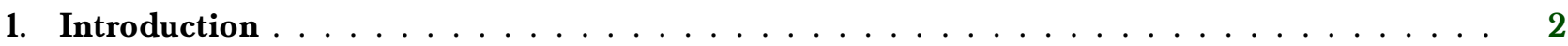

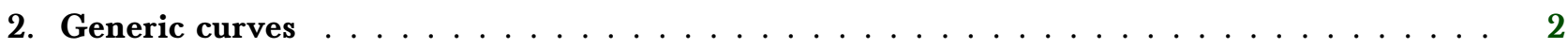

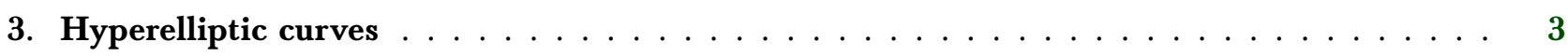

4. Examples of indecomposable limits $\ldots \ldots \ldots \ldots \ldots \ldots \ldots$

\section{Introduction}

Let $C$ be a smooth complex projective curve, and $E$ a vector bundle on $C$, of rank $r$. We will say that $E$ is a limit of $\mathcal{O}_{C}^{r}$ if there exists an algebraic family $\left(E_{b}\right)_{b \in B}$ of vector bundles on $C$, parametrized by an algebraic curve $B$, and a point $\mathrm{o} \in B$, such that $E_{\mathrm{o}}=E$ and $E_{b} \cong \mathcal{O}_{C}^{r}$ for $b \neq 0$. Can we classify all these vector bundles? If $E$ is a limit of $\mathcal{O}_{C}^{2}$ clearly $E \oplus \mathcal{O}_{C}^{r-2}$ is a limit of $\mathcal{O}_{C}^{r}$, so it seems reasonable to start in rank 2.

We get a complete classification in two extreme cases: when $C$ is generic (in the sense of Brill-Noether theory), and when it is hyperelliptic. In both cases the limit vector bundles are of the form $L \oplus L^{-1}$, with some precise conditions on $L$. However for large families of curves, for instance for plane curves, some limits of $\mathcal{O}_{C}^{2}$ are indecomposable, and those seem hard to classify.

\section{Generic curves}

Throughout the paper we denote by $C$ a smooth connected projective curve of genus $g$ over $\mathbb{C}$.

Proposition 1. Let $L$ be a line bundle on $C$ which is a limit of globally generated line bundles (in particular, any line bundle of degree $\geq g+1)$. Then $L \oplus L^{-1}$ is a limit of $\mathcal{O}_{C}^{2}$.

Proof. By hypothesis there exist a curve $B$, a point $\mathrm{o} \in B$ and a line bundle $\mathcal{L}$ on $C \times B$ such that $\mathcal{L}_{\mid C \times\{\mathrm{o}\}} \cong L$ and $\mathcal{L}_{\mid C \times\{b\}}$ is globally generated for $b \neq 0$. We may assume that $B$ is affine and that o is defined by $f=0$ for a global function $f$ on $B$; we put $B^{*}:=B \backslash\{\mathrm{o}\}$.

We choose two general sections $s, t$ of $\mathcal{L}$ on $C \times B^{*}$; reducing $B^{*}$ if necessary, we may assume that they generate $\mathcal{L}$. Thus we have an exact sequence on $C \times B^{*}$

$$
0 \rightarrow \mathcal{L}^{-1} \stackrel{(t,-s)}{\longrightarrow} \mathcal{O}_{C \times B^{*}}^{2} \stackrel{(s, t)}{\longrightarrow} \mathcal{L} \rightarrow 0
$$

which corresponds to an extension class $e \in H^{1}\left(C \times B^{*}, \mathcal{L}^{-2}\right)$. For $n$ large enough, $f^{n} e$ comes from a class in $H^{1}\left(C \times B, \mathcal{L}^{-2}\right)$ which vanishes along $C \times\{0\}$; this class gives rise to an extension

$$
0 \rightarrow \mathcal{L}^{-1} \longrightarrow \mathcal{E} \rightarrow \mathcal{L} \rightarrow 0
$$

with $\mathcal{E}_{\mid C \times\{b\}} \cong \mathcal{O}_{C}^{2}$ for $b \neq 0$, and $\mathcal{E}_{\mid C \times\{0\}} \cong L \oplus L^{-1}$.

Remark 1. Let $E$ be a vector bundle limit of $\mathcal{O}_{C}^{2}$. We have $\operatorname{det} E=\mathcal{O}_{C}$, and $h^{0}(E) \geq 2$ by semi-continuity. If $E$ is semi-stable this implies $E \cong \mathcal{O}_{C}^{2}$; otherwise $E$ is unstable. Let $L$ be the maximal destabilizing sub-line bundle of $E$; we have an extension $0 \rightarrow L \rightarrow E \rightarrow L^{-1} \rightarrow 0$, with $h^{0}(L) \geq 2$. Note that this extension is trivial (so that $E=L \oplus L^{-1}$ ) if $H^{1}\left(L^{2}\right)=0$, in particular if $\operatorname{deg}(L) \geq g$.

Proposition 2. Assume that $C$ is Brill-Noether-Petri general. The following conditions are equivalent:

(i) E is a limit of $\mathrm{O}_{C}^{2}$; 
(ii) $h^{0}(E) \geq 2$ and $\operatorname{det} E=\mathcal{O}_{C}$;

(iii) $E=L \oplus L^{-1}$ for some line bundle $L$ on $C$ with $h^{0}(L) \geq 2$ or $L=\mathcal{O}_{C}$.

Proof. We have seen that (i) implies (ii) (Remark 1). Assume (ii) holds, with $E \varsubsetneqq \mathcal{O}_{C}^{2}$. Then $E$ is unstable, and we have an extension $0 \rightarrow L \rightarrow E \rightarrow L^{-1} \rightarrow 0$ with $h^{0}(L) \geq 2$. Since $C$ is Brill-Noether-Petri general we have $H^{0}\left(C, K_{C} \otimes L^{-2}\right)=0$ [ACG, Ch. 21, Proposition 6.7], hence $H^{1}\left(C, L^{2}\right)=0$. Therefore the above extension is trivial, and we get (iii).

Assume that (iii) holds. Brill-Noether theory implies that any line bundle $L$ with $h^{0}(L) \geq 2$ is a limit of globally generated ones ${ }^{1}$. So (i) follows from Proposition 1.

\section{Hyperelliptic curves}

Proposition 3. Assume that $C$ is hyperelliptic, and let $H$ be the line bundle on $C$ with $h^{0}(H)=\operatorname{deg}(H)=2$. The limits of $\mathcal{O}_{C}^{2}$ are the decomposable bundles $L \oplus L^{-1}$, with $\operatorname{deg}(L) \geq g+1$ or $L=H^{k}$ for $k \geq 0$.

Proof. Let $\pi: C \rightarrow \mathbb{P}^{1}$ be the two-sheeted covering defined by $|H|$. Let us say that an effective divisor $D$ on $C$ is simple if it does not contain a divisor of the form $\pi^{*} p$ for $p \in \mathbb{P}^{1}$. We will need the following well-known lemma:

Lemma 1. Let L be a line bundle on $C$.

1) If $L=H^{k}(D)$ with D simple and $\operatorname{deg}(D)+k \leq g$, we have $h^{0}(L)=h^{0}\left(H^{k}\right)=k+1$.

2) If $\operatorname{deg}(L) \leq g, L$ can be written in a unique way $H^{k}(D)$ with $D$ simple. If $L$ is globally generated, it is a power of $H$.

Proof of Lemma 1. 1) Put $\ell:=g-1-k$ and $d:=\operatorname{deg}(D)$. Recall that $K_{C} \cong H^{g-1}$. Thus by Riemann-Roch, the first assertion is equivalent to $h^{0}\left(H^{\ell}(-D)\right)=h^{0}\left(H^{\ell}\right)-d$. We have $H^{0}\left(C, H^{\ell}\right)=\pi^{*} H^{0}\left(\mathbb{P}^{1}, \mathcal{O}_{\mathbb{P}^{1}}(\ell)\right)$; since $D$ is simple of degree $\leq \ell+1$, it imposes $d$ independent conditions on $H^{0}\left(C, H^{\ell}\right)$, hence our claim.

2) Let $k$ be the greatest integer such that $h^{0}\left(L \otimes H^{-k}\right)>0$; then $L=H^{k}(D)$ for some effective divisor $D$, which is simple since $k$ is maximal. By 1$) D$ is the fixed part of $|L|$, hence is uniquely determined, and so is $k$. In particular the only globally generated line bundles on $C$ of degree $\leq g$ are the powers of $H$.

Proof of the Proposition: Let $E$ be a vector bundle on $C$ limit of $\mathcal{O}_{C}^{2}$. Consider the exact sequence

$$
0 \rightarrow L \rightarrow E \rightarrow L^{-1} \rightarrow 0
$$

where we can assume $\operatorname{deg}(L) \leq g$ (Remark 1). By Lemma 1 we have $L=H^{k}(D)$ with $D$ simple of degree $\leq g-2 k$. After tensor product with $H^{k}$, the corresponding cohomology exact sequence reads

$$
0 \rightarrow H^{0}\left(C, H^{2 k}(D)\right) \rightarrow H^{0}\left(C, E \otimes H^{k}\right) \rightarrow H^{0}\left(C, \mathcal{O}_{C}(-D)\right) \stackrel{\partial}{\longrightarrow} H^{1}\left(C, H^{2 k}(D)\right)
$$

which implies $h^{0}\left(E \otimes H^{k}\right)=h^{0}\left(H^{2 k}(D)\right)+\operatorname{dim} \operatorname{Ker} \partial=2 k+1+\operatorname{dim} \operatorname{Ker} \partial$ by Lemma 1 .

By semi-continuity we have $h^{0}\left(E \otimes H^{k}\right) \geq 2 h^{0}\left(H^{k}\right)=2 k+2$; the only possibility is $D=0$ and $\partial=0$. But $\partial(1)$ is the class of the extension (1), which must therefore be trivial; hence $E=H^{k} \oplus H^{-k}$.

$1 \uparrow$ Indeed, the subvariety $W_{d}^{r}$ of $\operatorname{Pic}^{d}(C)$ parametrizing line bundles $L$ with $h^{0}(L) \geq r+1$ is equidimensional, of dimension $g-(r+1)(r+g-d)$; the line bundles which are not globally generated belong to the subvariety $W_{d-1}^{r}+C$, which has codimension $r$. 


\section{Examples of indecomposable limits}

To prove that some limits of $\mathcal{O}_{C}^{2}$ are indecomposable we will need the following easy lemma:

Lemma 2. Let $L$ be a line bundle of positive degree on $C$, and let

$$
0 \rightarrow L \rightarrow E \rightarrow L^{-1} \rightarrow 0
$$

be an exact sequence. The following conditions are equivalent:

(i) E is indecomposable;

(ii) The extension (2) is nontrivial;

(iii) $h^{0}(E \otimes L)=h^{0}\left(L^{2}\right)$.

Proof. The implication (i) $\Rightarrow$ (ii) is clear.

(ii) $\Rightarrow$ (iii) : After tensor product with $L$, the cohomology exact sequence associated to (2) gives

$$
0 \rightarrow H^{0}\left(L^{2}\right) \stackrel{i}{\longrightarrow} H^{0}(E \otimes L) \longrightarrow H^{0}\left(\mathcal{O}_{C}\right) \stackrel{\partial}{\longrightarrow} H^{1}\left(L^{2}\right),
$$

where $\partial$ maps $1 \in H^{0}\left(\mathcal{O}_{C}\right)$ to the extension class of (2). Thus (ii) implies that $i$ is an isomorphism, hence (iii).

(iii) $\Rightarrow$ (i): If $E$ is decomposable, it must be equal to $L \oplus L^{-1}$ by unicity of the destabilizing bundle. But this implies $h^{0}(E \otimes L)=h^{0}\left(L^{2}\right)+1$.

The following construction was suggested by N. Mohan Kumar:

Proposition 4. Let $C \subset \mathbb{P}^{2}$ be a smooth plane curve, of degree d. For $0<k<\frac{d}{4}$, there exist extensions

$$
0 \rightarrow \mathcal{O}_{C}(k) \rightarrow E \rightarrow \mathcal{O}_{C}(-k) \rightarrow 0
$$

such that $E$ is indecomposable and is a limit of $\mathcal{O}_{C}^{2}$.

Proof. Let $Z$ be a finite subset of $\mathbb{P}^{2}$ which is the complete intersection of two curves of degree $k$, and such that $C \cap Z=\varnothing$. By [S, Remark 4.6], for a general extension

$$
0 \rightarrow \mathcal{O}_{\mathbb{P}^{2}}(k) \rightarrow E \rightarrow \mathcal{I}_{Z}(-k) \rightarrow 0,
$$

the vector bundle $E$ is a limit of $\mathcal{O}_{\mathbb{P}^{2}}^{2}$; therefore $E_{\mid C}$ is a limit of $\mathcal{O}_{C}^{2}$.

The extension (3) restricts to an exact sequence

$$
0 \rightarrow \mathcal{O}_{C}(k) \rightarrow E_{\mid C} \rightarrow \mathcal{O}_{C}(-k) \rightarrow 0
$$

To prove that $E_{\mid C}$ is indecomposable, it suffices by Lemma 2 to prove that $h^{0}\left(E_{\mid C}(k)\right)=h^{0}\left(\mathcal{O}_{C}(2 k)\right)$. Since $2 k<d$ we have $h^{0}\left(\mathcal{O}_{C}(2 k)\right)=h^{0}\left(\mathcal{O}_{\mathbb{P}^{2}}(2 k)\right)=h^{0}(E(k))$, so in view of the exact sequence

$$
0 \rightarrow E(k-d) \longrightarrow E(k) \longrightarrow E_{\mid C}(k) \rightarrow 0
$$

it suffices to prove $H^{1}(E(k-d))=0$, or by Serre duality $H^{1}(E(d-k-3))=0$.

The exact sequence (3) gives an injective map $H^{1}(E(d-k-3)) \hookrightarrow H^{1}\left(\mathcal{I}_{Z}(d-2 k-3)\right)$. Now since $Z$ is a complete intersection we have an exact sequence

$$
0 \rightarrow \mathcal{O}_{\mathbb{P}^{2}}(-2 k) \rightarrow \mathcal{O}_{\mathbb{P}^{2}}(-k)^{2} \rightarrow \mathcal{I}_{Z} \rightarrow 0 ;
$$

since $4 k<d$ we have $H^{2}\left(\mathcal{O}_{\mathbb{P}^{2}}(d-4 k-3)\right)=0$, hence $H^{1}\left(\mathcal{I}_{Z}(d-2 k-3)\right)=0$, and finally $H^{1}(E(d-k-3))=0$ as asserted. 
We can also perform the Strømme construction directly on the curve $C$, as follows. Let $L$ be a base point free line bundle on $C$. We choose sections $s, t \in H^{0}(L)$ with no common zero. This gives rise to a Koszul extension

$$
0 \rightarrow L^{-1} \stackrel{i}{\longrightarrow} \mathcal{O}_{C}^{2} \stackrel{p}{\longrightarrow} L \rightarrow 0 \quad \text { with } i=(-t, s), p=(s, t) .
$$

We fix a nonzero section $u \in H^{0}\left(L^{2}\right)$. Let $\mathcal{L}$ be the pull-back of $L$ on $C \times \mathbb{A}^{1}$. We consider the complex ("monad")

$$
\mathcal{L}^{-1} \stackrel{\alpha}{\longrightarrow} \mathcal{L}^{-1} \oplus \mathcal{O}^{2} \oplus \mathcal{L} \stackrel{\beta}{\longrightarrow} \mathcal{L}, \quad \alpha=(\lambda, i, u), \beta=(u, p,-\lambda),
$$

where $\lambda$ is the coordinate on $\mathbb{A}^{1}$. Let $\mathcal{E}:=\operatorname{Ker} \beta / \operatorname{Im} \alpha$, and let $E:=\mathcal{E}_{\mid C \times\{0\}}$.

Lemma 3. $E$ is a rank 2 vector bundle, limit of $\mathcal{O}_{C}^{2}$. There is an exact sequence $0 \rightarrow L \rightarrow E \rightarrow L^{-1} \rightarrow 0$; the corresponding extension class in $H^{1}\left(L^{2}\right)$ is the product by $u^{2} \in H^{0}\left(L^{4}\right)$ of the class $e \in H^{1}\left(L^{-2}\right)$ of the Koszul extension (4).

Proof. The proof is essentially the same as in [S]; we give the details for completeness.

For $\lambda \neq 0$, we get easily $\mathcal{E}_{\mid C \times\{\lambda\}} \cong \mathcal{O}_{C}^{2}$; we will show that $E$ is a rank 2 vector bundle. This implies that $\mathcal{E}$ is a vector bundle on $C \times \mathbb{A}^{1}$, and therefore that $E$ is a limit of $\mathcal{O}_{C}^{2}$.

Let us denote by $\alpha_{0}, \beta_{0}$ the restrictions of $\alpha$ and $\beta$ to $C \times\{0\}$. We have $\operatorname{Ker} \beta_{0}=L \oplus N$, where $N$ is the kernel of $(u, p): L^{-1} \oplus \mathcal{O}_{C}^{2} \rightarrow L$. Applying the snake lemma to the commutative diagram

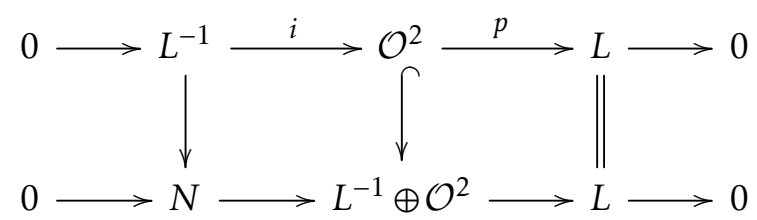

we get an exact sequence

$$
0 \rightarrow L^{-1} \rightarrow N \rightarrow L^{-1} \rightarrow 0
$$

which fits into a commutative diagram

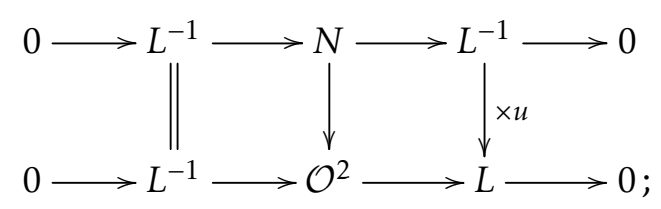

this means that the extension (5) is the pull-back by $\times u: L^{-1} \rightarrow L$ of the Koszul extension (4).

Now since $E$ is the cokernel of the map $L^{-1} \rightarrow L \oplus N$ induced by $\alpha_{0}$, we have a commutative diagram

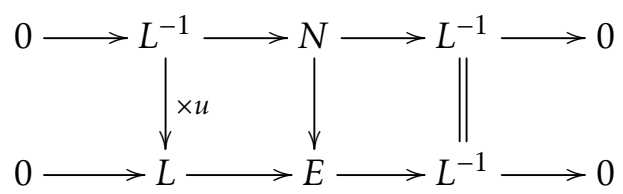

so that the extension $L \rightarrow E \rightarrow L^{-1}$ is the push-forward by $\times u$ of (5). This implies the Lemma.

Unfortunately it seems difficult in general to decide whether the extension $L \rightarrow E \rightarrow L^{-1}$ nontrivial. Here is a case where we can conclude:

Proposition 5. Assume that $C$ is non-hyperelliptic. Let $L$ be a globally generated line bundle on $C$ such that $L^{2} \cong K_{C}$. Let $0 \rightarrow L \rightarrow E \rightarrow L^{-1} \rightarrow 0$ be the unique nontrivial extension of $L^{-1}$ by $L$. Then $E$ is indecomposable, and is a limit of $\mathcal{O}_{C}^{2}$. 
Proof. We choose $s, t$ in $H^{0}(L)$ without common zero, and use the previous construction. It suffices to prove that we can choose $u \in H^{0}\left(K_{C}\right)$ so that $u^{2} e \neq 0$ : since $H^{1}\left(K_{C}\right) \cong \mathbb{C}$, the vector bundle $E$ will be the unique nontrivial extension of $L^{-1}$ by $L$, and indecomposable by Lemma 2 .

Suppose that $u^{2} e=0$ for all $u$ in $H^{0}\left(K_{C}\right)$; by bilinearity this implies $u v e=0$ for all $u, v$ in $H^{0}\left(K_{C}\right)$. Since $C$ is not hyperelliptic, the multiplication map $\mathrm{S}^{2} H^{0}\left(K_{C}\right) \rightarrow H^{0}\left(K_{C}^{2}\right)$ is surjective, so we have we=0 for all $w \in H^{0}\left(K^{2}\right)$. But the pairing

$$
H^{1}\left(K_{C}^{-1}\right) \otimes H^{0}\left(K_{C}^{2}\right) \rightarrow H^{1}\left(K_{C}\right) \cong \mathbb{C}
$$

is perfect by Serre duality, hence our hypothesis implies $e=0$, a contradiction.

Remark 2. In the moduli space $\mathcal{M}_{g}$ of curves of genus $g \geq 3$, the curves $C$ admitting a line bundle $L$ with $L^{2} \cong K_{C}$ and $h^{0}(L)$ even $\geq 2$ form an irreducible divisor [T2]; for a general curve $C$ in this divisor, the line bundle $L$ is unique, globally generated, and satisfies $h^{0}(L)=2$ [T1]. Thus Proposition 5 provides for $g \geq 4$ a codimension 1 family of curves in $\mathcal{M}_{g}$ admitting an indecomposable vector bundle limit of $\mathcal{O}_{C}^{2}$.

Remark 3. Let $\pi: C \rightarrow B$ be a finite morphism of smooth projective curves. If $E$ is a vector bundle limit of $\mathcal{O}_{B}^{2}$, then clearly $\pi^{*} E$ is a limit of $\mathcal{O}_{C}^{2}$. Now if $E$ is indecomposable, $\pi^{*} E$ is also indecomposable. Consider indeed the nontrivial extension $0 \rightarrow L \rightarrow E \rightarrow L^{-1} \rightarrow 0$ (Remark 1); by Lemma 2 it suffices to show that the class $e \in H^{1}\left(B, L^{2}\right)$ of this extension remains nonzero in $H^{1}\left(C, \pi^{*} L^{2}\right)$. But the pull-back homomorphism $\pi^{*}: H^{1}\left(B, L^{2}\right) \rightarrow H^{1}\left(C, \pi^{*} L^{2}\right)$ can be identified with the homomorphism $H^{1}\left(B, L^{2}\right) \rightarrow H^{1}\left(B, \pi_{*} \pi^{*} L^{2}\right)$ deduced from the linear map $L^{2} \rightarrow \pi_{*} \pi^{*} L^{2}$, and the latter is an isomorphism onto a direct factor; hence $\pi^{*}$ is injective and $\pi^{*} e \neq 0$, so $E$ is indecomposable.

Thus any curve dominating one of the curves considered in Propositions 4 and 5 carries an indecomposable vector bundle which is a limit of $\mathcal{O}_{C}^{2}$.

\section{References}

[ACGH] E. Arbarello, M. Cornalba, P.A. Griffiths, and J. Harris, Geometry of algebraic curves. Vol. I, Grundlehren der Mathematischen Wissenschaften, vol. 267, Springer-Verlag, New York, 1985. MR0770932

[ACG] E. Arbarello, M. Cornalba, and P.A. Griffiths, Geometry of algebraic curves. Vol. II, with a contribution by Joseph Daniel Harris, Grundlehren der Mathematischen Wissenschaften, vol. 268, Springer, Heidelberg, 2011. MR-2807457

[H] J. Harris, Theta-characteristics on algebraic curves, Trans. Amer. Math. Soc. 271 (1982), no. 2, 611638. MR-0654853

[S] S. A. Strømme, Deforming vector bundles on the projective plane, Math. Ann. 263 (1983), no. 3, 385-397. MR-0704303

[T1] M. Teixidor i Bigas, Half-canonical series on algebraic curves, Trans. Amer. Math. Soc. 302 (1987), no. 1, 99-115. MR-0887499

[T2] M. Teixidor i Bigas, The divisor of curves with a vanishing theta-null, Compositio Math. 66 (1988), no. 1, 15-22. MR-0937985 